\section{Targeted agents reverse chemotherapy resistance in urothelial carcinoma}

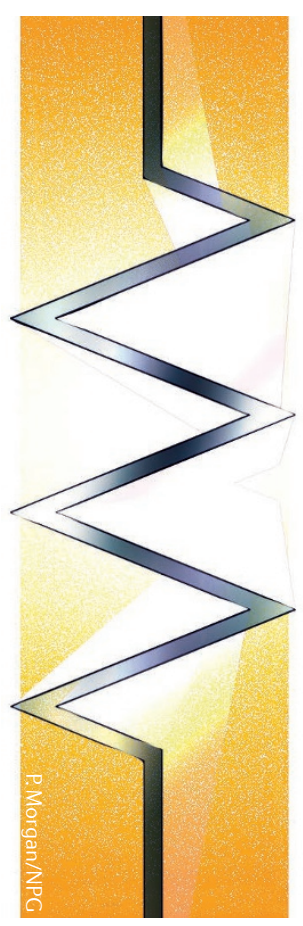

Patients with urothelial carcinoma of the bladder (UCB) typically receive cisplatin, often in combination with paclitaxel; however, some patients are intrinsically resistant to these agents and others will acquire resistance, both of which can result in treatment failure. Now, newly published research has shown that exposure to the epidermal growth factor receptor (EGFR) inhibitor gefitinib, or the transducer and activator of transcription 3 (STAT3) inhibitor S3I-201 enhances the sensitivity of UCB cells to cisplatin and paclitaxel.

Investigators conducted in vitro investigations using cisplatinresistant UCB cell lines to explore the potential to modulate the effects of the transcription factor CCAAT/enhancer-binding protein $\delta$ (CEBPD). Lead author Ju-Ming Wang explains: "according to our previous findings, CEBPD has a critical role in cisplatin-resistant UCB;" therefore, "we investigated several aspects including how CEBPD is upregulated in response to CDDPinduced chemotherapy resistance, whether CEBPD has a functional role in cross-resistance to paclitaxel in cisplatin-resistant UCB, and how to overcome resistance to chemotherapy in UCB cells".

After confirming that exposure to cisplatin and paclitaxel upregulates CEBPD expression, the researchers found that increased CEBPD expression upregulates multidrug resistance protein 1 (ABCB1) drug efflux transporter expression, and that CEBPD knockdown attenuates this effect. Researchers also observed an increase in EGFR and STAT3 expression following exposure to cisplatin and confirmed the importance of this expression by demonstrating that S3I-201 inhibits either cisplatin-induced or EGF-induced upregulation of CEBPD. Furthermore, both gefitinib and S3I-201 attenuated ABCB1-mediated drug efflux, resulting in cisplatin-induced or paclitaxelinduced cytotoxicity in previously resistant cells.

These in vitro findings were confirmed in mice with tumours induced by UCB-cell injection: mice treated with cisplatin plus either S3I-201 or gefitinib had tumours of significantly reduced size and weight, compared with cisplatin-treated controls.

Wang summarizes "inhibition of the EGFR/STAT3 signalling pathway could provide a solution to the problem of therapeutic resistance to chemotherapy in patients with UCB" and on the future directions of this research, adds: "We are interested in continuing to explore the tumour-promoting role of CEBPD in UCB and other cancer types."

Peter Sidaway

ORIGINAL ARTICLE Wang, W. J. et al. Inhibition of EGFR/STAT3/CEBPD axis reverses cisplatin cross-resistance with paclitaxel in urothelial carcinoma of urinary bladder. Clin. Cancer Res. http://dx.doi.org/10.1158/1078-0432.CCR-15$\underline{1169}$ (2016) 